\title{
Application of Computational Intelligence Methods for Intelligent Modelling of Buildings
}

\author{
Alexander Gegov \\ Department of Computer Science and Software Engineering, \\ University of Portsmouth, United Kingdom, e-mail: alexander.gegov@port.ac.uk
}

\begin{abstract}
This paper considers the application of soft computing techniques for predictive modelling in the built sector and presents the extention of the results from previous works of the author. While the latter considers only short-term modelling which is used mainly for control purposes, the present paper discusses also long-term modelling results that may be used for efficiency evaluation in buildings. Three different types of buildings are considered, an air-conditioned zone, a naturally ventilated room, and an endothermic building. The are subjected to their normal occupancy effects and the natural external climatic disturbances which are difficult to incorporate in accurate modelling using conventional quantitative methods. The approach adopted here uses fuzzy logic for modelling, as well as neural networks and genetic algorithms for adaptation and optimisation of the fuzzy model. Takagi-Sugeno fuzzy models are built by subtractive clustering to provide initial values of the antecedent non-linear membership functions parameters and the consequent linear algebraic equations coefficients. A method of extensive searching the possible solution space is presented which explores all the possible permutations for a specified range of orders to derive the initial fuzzy model. This model is an extension of the traditional ARMAX (Auto Regressive Moving Average Exogenous) model where the effect of the moving average term has been accounted for by the fuzziness and its ability to represent uncertainty. The fuzzy model parameters are further adjusted by a back-propagation neural network and a real-valued genetic algorithm in order to obtain a better fit to the measured data. Model validation results using data from the three buildings are presented where the initial (fuzzy) and the improved (fuzzy-neuro and fuzzygenetic) models are compared and analysed with each other and with conventional (non-fuzzy) models.
\end{abstract}

Keywords. Fuzzy logic, fuzzy modelling, neural networks, model adaptation, genetic algorithms, genetic optimisation. 


\section{Introduction}

Soft Computing (SC) is a heuristic methodology which has attracted significant interest in recent years and has shown to be successful in many areas such as modelling, control, fault diagnosis and pattern recognition. It is based on the cooperative implementation of different approaches such as Fuzzy Logic (FL), Neural Networks (NN), and Genetic Algorithms (GA) [5, 6]. Each of these techniques is suited for solving specific types of problems. In this respect, FL is powerful for approximate reasoning and logical inference-based modelling, $\mathrm{NN}$ are well suited for learning-based adaptation while GA are efficient for evolution-based optimisation. The underlying idea of SC is to use these heuristic approaches in synergetic way with each other as well as combining them with conventional approaches, rather than using each of them separately.

The research presented here is aimed at developing efficient control methods to regulate the internal climates in buildings. The authors develop good quality models which can enable proactive control strategies to be developed in preference to the currently used reactive ones. In other words, instead of applying a control action only on the basis of the currently measured sensor values, the concept is to take into account future model based predictions of thee values as well. The advantage of such a proactive control philosophy lies in the possibility to apply heating and cooling control efforts more efficiently as a result of which the variations of the controlled parameters become smoother as well as better regulation performance is possible. This will lead to lower energy consumptions and reduced environmental pollution. However, obtaining predictive models for these buildings is not a trivial task because the thermal behaviour is affected by climatic and occupancy effects which are characterised by significant complexity and uncertainty as already mentioned.

The notion of a proactive control philosophy is the following: the control action to be applied at the current time instant $k$ is computed not only on the basis of measured variables at the time $k$ and the past $(k-1),(k-2)$, etc, but also by taking into account of predictions at the future time instants $(k+1),(k+2)$, etc.

It would be interesting to see, if the SC methodology can provide models of sufficient quality for the built environment so that such proactive model based strategies can be developed. Some investigations have recently been carried out in this domain but they have been narrow and have lead to limited conclusions. In most cases, they are focused on one modelled parameter and make use of individual FL, NN and GA techniques, rather than adapting a coordinated SC methodology. In other words, the potential of the SC methodology for buildings has still not been explored.

This paper presents results from a research project aimed at investigating the capabilities of the SC methodology for predictive modelling the temperature and relative humidity in buildings. The results are based on preliminary works of the research team $[1,2,3,4,7]$. Three types of buildings are considered in the study, namely: air conditioned, naturally ventilated and endothermic buildings. The three specific buildings under investigation are: the Anglesea Building (University of 
Portsmouth), the Portland Building (University of Porsmouth) and St Catherine's Lighthouse (Trinity House, Isle of Wight). These buildings differ substantially and the intention is to identify how each type is suited to benefit from the SC methodology.

\section{Methodology}

The approach adopted uses Takagi-Sugeno (TS) fuzzy models which have received considerable attention over the last 10 years because of their suitability for processing information from input-output measurements [6]. In BMS applications, the main information source is the measured data from sensor readings making TS FL models easily realisable rather than having to rely on expert knowledge which is difficult to obtain because of the complex multivariable nature of the application [6]. Another advantage of the TS fuzzy model is its capability to approximate nonlinear input-output mappings by a number of linearised models at a number of suitable operating points.

The TS fuzzy model consists of linguistic if-then rules in the antecedent part and linear algebraic equations in the consequent part. There are two types of parameters in this model: non-linear ones in the membership functions in the antecedent part and linear ones in the algebraic equations in the consequent part. The initial fuzzy model is obtained by determining the values of both parameters on the basis of the input-output data. The method used in the paper is based on subtractive clustering, i.e. by assuming that each data point is a potential cluster centre and using all the data to gradually find the final clustering.

The Takagi-Sugeno fuzzy model for a system with two rules, two inputs $\left(u_{1}, u_{2}\right)$ and one output $(y)$ is presented by Equation (1). The linguistic labels (membership functions) of the inputs are denoted by $A_{i}, B_{i}, i=1,2$ and their parameters are the non-linear antecedent parameters. The coefficients $a_{i}, b_{i}, i=1,2,3$ are the linear consequent parameters used for the computation of the output.

$$
\begin{aligned}
& \text { If } u_{1} \text { is } A_{1} \text { and } u_{2} \text { is } A_{2} \text { then } y=a_{1} \cdot u_{1}+a_{2} \cdot u_{2}+a_{3} \\
& \text { If } u_{1} \text { is } B_{1} \text { and } u_{2} \text { is } B_{2} \text { then } y=b_{1} \cdot u_{1}+b_{2} \cdot u_{2}+b_{3}
\end{aligned}
$$

Equation (1) represents a static Takagi-Sugeno fuzzy model which does not contain the time argument. However, in order to dynamically predict the temperature and the relative humidity, it should be included in the equation, i.e. the model must be a dynamic one. In this respect, two types of models are introduced and investigated here, namely Regression Delay (RD) and Proportional Difference (PD). Examples of such models are represented by Equations (2) and (3), respectively.

$$
\begin{aligned}
& \text { If } y_{k} \text { is } A_{1} \text { and } y_{k-1} \text { is } A_{2} \text { and } u_{1, k} \text { is } A_{3} \text { and } u_{1, k-1} \text { is } A_{4} \text { and } u_{2, k-1} \text { is } A_{5} \\
& \text { then } y_{k+1}=a_{1} \cdot y_{k}+a_{2} \cdot y_{k-1}+a_{3} \cdot u_{1, k}+a_{4} \cdot u_{1, k-1}+a_{5} \cdot u_{2, k-1}+a_{6}
\end{aligned}
$$

IIf $y_{k}$ is $A_{1}$ and $D y_{k}$ is $A_{2}$ and $u_{1, k}$ is $A_{3}$ and $D u_{2, k}$ is $A_{4}$

then $\mathrm{y}_{\mathrm{k}+1}=\mathrm{a}_{1} \cdot \mathrm{y}_{\mathrm{k}}+\mathrm{a}_{2} \cdot D \mathrm{y}_{\mathrm{k}}+\mathrm{a}_{3} \cdot \mathrm{u}_{1, \mathrm{k}}+\mathrm{a}_{4} \cdot D \mathrm{u}_{2, \mathrm{k}}+\mathrm{a}_{5}$

where $D y_{k}=y_{k}-y_{k-1}, D u_{2, k}=u_{2, k}-u_{2, k-1}$ 
It can be seen that Equation (2) contains two auto-regression terms of the output $y$, two regression terms of the input $u_{1}$ and one delay term for the input $u_{2}$. In contrast, Equation (3) contains one proportional and one difference term of the output $y$, one proportional term of the input $u_{1}$ and one difference term of the input $u_{2}$. For simplicity purposes, each of the equations includes only one rule, but in general the number of rules is higher; it is equal to the number of the linearised sub-models applicable to the respective local regions of the whole operating range.

Equations (2)-(3) represent examples of fuzzy model structures. Usually, these structures are obtained on the basis of evaluating a number of model structures in accordance with a performance criterion (usually the Root Mean Squared Error RMSE). The latter is a measure of the closeness of the model to the plant and is expressed by Equation (4).

Equations (2)-(3) represent examples of fuzzy model structures. Usually, these structures are obtained on the basis of evaluating a number of model structures in accordance with a performance criterion (usually the Root Mean Squared Error RMSE). The latter is a measure of the closeness of the model to the plant and is expressed by Equation (4).

$$
\operatorname{RMSE}=\left[\left(\mathrm{y}_{1}^{\mathrm{P}}-\mathrm{y}_{1}{ }_{1}\right)^{2}+\ldots+\left(\mathrm{y}^{\mathrm{P}}{ }_{\mathrm{N}}-\mathrm{y}^{\mathrm{M}}{ }_{\mathrm{N}}\right)^{2}\right] / \mathrm{N}
$$

where $N$ is the number of data points, and the superscripts $P$ and $M$ stand for the plant and the model, respectively.

The majority of identification techniques apply partial rather than extensive model structural searching to obtain the best structures. Usually, the approach is to represent the plant dynamics by either regression or delay terms. As far as proportional or difference terms are concerned, they are usually considered up to the most recent data point in the past. This might be a serious disadvantage as the dynamics of the plant are not fully explored and some important terms in the model structure may remain unaccounted for giving non-optimal results.

This paper presents a method of searching the range of model structures so that all the possible permutations of a specified order are explored. This method is demonstrated here for Takagi-Sugeno fuzzy models but can be also applied to any other types of models, including traditional ones. For a plant with $m$ input variables and backward horizon equal to $b$, the whole number of investigated model structures $\mathrm{S}$ is given by Equation (5).

$$
\mathrm{S}=(2 . \mathrm{b})^{\mathrm{m}}-1
$$

It is evident from Equation (5) that the number of investigated models is an exponential function of the number of inputs and the backward horizon. This can lead to long processing because of the extensive searching involved. However, this is important to do as getting the model structure right is crucial to the success of the whole modeling procedure.

The task of neural adaptation is to adjust the fuzzy model parameters in order to obtain a better fit to the measured data. The method used in the paper is based on the idea of back-propagation, i.e. by iteratively propagating of the model error (the difference between the real and the modelled plant output) from the consequent to 
the antecedent part of the fuzzy rules until a specified number of iterations is achieved [6].

The main options for the neural network adaptation are the number of iterations, the error goal, the initial step size, the decreasing and the increasing learning rates. The number of iterations gives the number of new sets of parameter values evaluated, the error goal is a measure for the desired model accuracy, the initial step size shows the magnitude of change in the initial parameter values, while the decreasing/increasing learning rates take into account the negative/positive changes in the model's accuracy in the last iteration in comparison to the previous one.

The purpose of genetic optimisation is to adjust the model parameters in an alternative manner; the method used here is based on the idea of real-valued coding, i.e., by representing the individuals with real-valued genes and sequentially evaluating the modelled error until a pre-specified number of generations is reached [5].

\section{Results and discussion}

This section presents modelling results for the three buildings under consideration over the heating season. The results show that the outputs (temperature and relative humidity) can be accurately predicted on the basis of measurements from the buildings using SC modelling techniques. The variables are chosen to be the temperatures and relative humidities in the same and neighbouring zones. For the purpose of systematic modelling, both RD and PD types of fuzzy models have been considered. The best model structure corresponding to the smallest prediction error was chosen by evaluating all possible structures of models for order 2, i.e. involving all combinations of up to two input terms. Afterwards, the quality of the models was evaluated by using a new data set and the respective validation error was obtained. The errors calculated were both one-step ahead predictions and longer-term forecasts. The membership functions of the inputs in the fuzzy model were chosen to be of Gaussian types while the selected options for the neural network and the genetic algorithm were 500 iterations and 50 generations with 10 individuals each, respectively. These options seem to give a fair comparison between the two considered fuzzy model improvement techniques as the number of investigated fuzzy membership function parameters is equal to 500 in both cases.

Each one of the above model structures was chosen from a set of 1023 possible models, representing all combinations of (auto-) regressional and (auto-)delayed terms. The backward (dynamical memory) horizon was chosen to be 2, i.e., the prediction of the internal temperature at time $k+1$ is obtained on the basis of measurements of two previous values, namely, at times $k$ and $k-1$. The plant and the model outputs for each of these one-step ahead prediction models and for each building are shown in figs 1-3. In the longer-term horizon case, the respective model root mean square errors are not shown in the paper due the limited space available. 

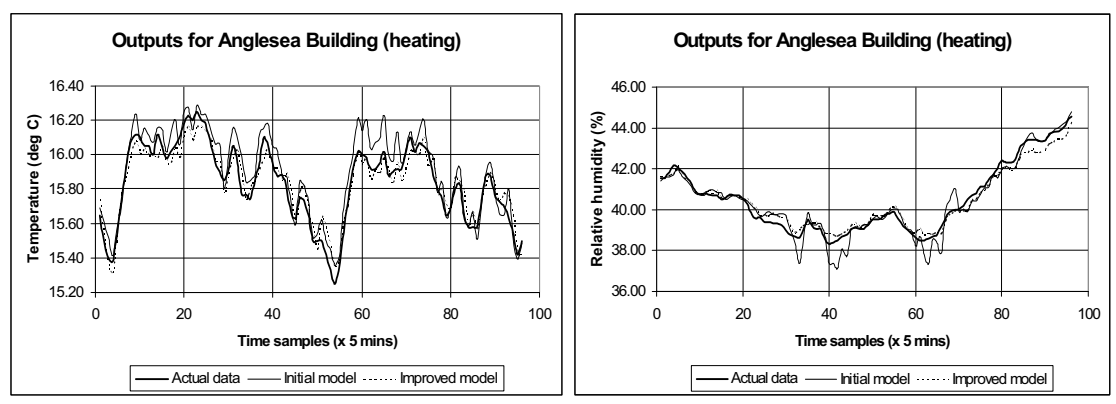

Fig. 1. Temperature and relative humidity outputs for Anglesea Bulding.
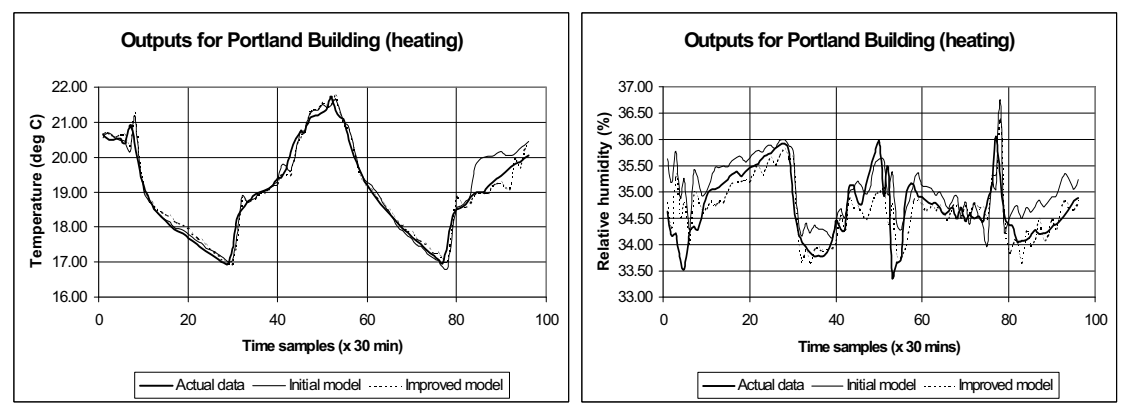

Fig. 2. Temperature and relative humidity outputs for Portland Bulding.
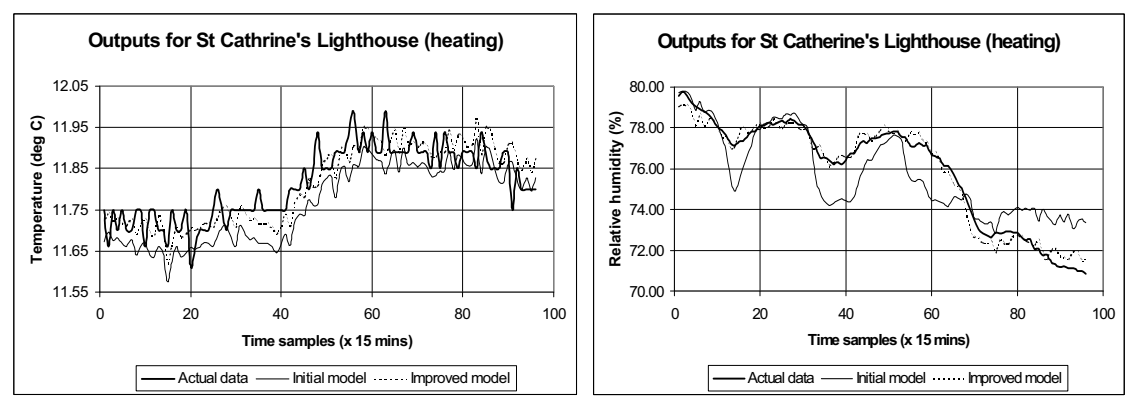

Fig. 3. Temperature and relative humidity outputs for St Catherine's Lighthouse.

It is evident for Anglesea Building and St Catherine's Lighthouse that the best model structures are of a regression-delay type, while for Portland Building they are of a proportional-difference type. 


\section{Conclusion}

The best prediction results have been obtained for the naturally ventilated area, while the results for the air-conditioned zone and the endothermic building are not as good but comparable to each other. The temperature prediction is better than the humidity prediction for the three buildings because of the smaller variation range and smoother profile. The proportional-difference fuzzy model performs better than the regression-delay model in most cases for all the buildings.

In most cases, when the one-step ahead prediction of the fuzzy model is significantly improved by neural networks and/or genetic algorithms, the long-term prediction results are also improved. The long-term prediction error obtained is usually comparable to or is even bigger than that obtained before adaptation/optimisation of the fuzzy model. It is interesting to point out that in some cases the long term prediction error is smaller than the one-step ahead error either before or after model improvement. This happens in the case of Potland Building and is possibly due to the cyclic behaviour of the building which makes it possible for the model to predict more distant future values of temperatures and relative humidities very well because these values are close to some of the previous values of these two internal variables.

In general, there is a substantial improvement of the prediction capabilities of the initial fuzzy model for both short and long term prediction intervals, especially in the cases when this model is not very good. In this respect, genetic algorithms are in most cases superior to neural networks for both temperature and humidity predictions, for both types of regression-delay and proportional-difference fuzzy models (for all three building types). Genetic algorithms seem to show considerable better convergence properties than neural networks as the best model parameters are usually obtained from the last genetic generations while these parameters are in most cases found from the first neural iterations.

\section{References}

1. Azzi, D., Gegov, A., Virk, G., Haynes, B., and Alkadhimi, K. (2000) Application of Soft-computing Techniques in Modeling of Buildings, International Workshop on Recent Advances in Soft Computing, Leicester, UK, vol.1, pp. 143-150.

2. Gegov, A., Virk, G., Azzi, D., Haynes, B., and Alkadhimi, K. (2001) Soft-Computing Based Predictive Modelling of Building Management Systems, International Journal of Knowledge Based Intelligent Engineering Systems, vol.5, no.1, pp.41-51.

3. Gegov, A., Virk, G., Azzi, D., Haynes, B., and Alkadhimi, K. (1999) Soft-Computing Based Predictive Modelling of Building Management Systems, International Workshop on Recent Advances in Soft Computing, Leicester, UK, vol.1, pp.69-77.

4. Gegov, A., Virk, G., Azzi, D., Haynes, B., and Alkadhimi, K. (1999) Soft-Computing Based Modelling of the Internal Climate in Office Buildings, UK Workshop on Fuzzy Systems, Uxbridge, UK, vol.1, pp.145-152. 
5. Haupt, R., and Haupt, S. (1998) Practical Genetic Algorithms, John Wiley \& Sons, New York.

6. Jang, J., Sun, C., and Mizutani, E. (1997) Neuro-Fuzzy and Soft Computing, Prentice Hall, Upper Saddle River.

7. Virk, G., Azzi, D., Gegov, A., Haynes, B., and Alkadhimi, K. (2003) Intelligent SoftComputing Based Modelling of Naturally Ventilated Buildings, International Journal of Solar Energy, to appear. 